\title{
Energetics and electronic structures of single walled carbon nanotubes encapsulated in boron nitride nanotubes
}

\author{
Kaoru Hisama ${ }^{1}$, Shohei Chiashi, ${ }^{1}$, Shigeo Maruyama ${ }^{1,3}$ and Susumu Okada ${ }^{2}$ \\ ${ }^{1}$ Department of Mechanical Engineering, The University of Tokyo, Tokyo 113-8656, Japan \\ ${ }^{2}$ Graduate School of Pure and Applied Sciences, University of Tsukuba, Tsukuba 305-8571, Japan \\ ${ }^{3}$ Energy NanoEngineering Lab., National Institute of Advanced Industrial Science and Technology \\ (AIST), Tsukuba 305-8564, Japan
}

\begin{abstract}
Using the density functional theory, energetics and electronic structures of single walled carbon nanotube (CNT) encapsulated in boron nitride nanotube (BNNT) are investigated. Cohesive energy of CNT inside BNNT depends on the spacing between them and on their mutual arrangement. Band gap of CNT encapsulated in BNNT is modulated by about tens of meV, when they have $A B$ stacking arrangement where $B$ atoms are located as close as to $C$ atoms, indicating that BNNT effectively affects the tensile strain onto CNT. Carrier accumulation into CNT occurs under external electric field, where BNNT acts as the gate insulator surrounding CNT.
\end{abstract}

Ever since the discovery of a tubular form of $\mathrm{sp}^{2} \mathrm{C}$ atoms, ${ }^{1)}$ carbon nanotubes (CNTs), they have been keeping a premier position in fields of nanoscale sciences and technologies. ${ }^{2)}$ Honeycomb covalent networks of $\mathrm{C}$ atoms with the cylindrical boundary condition endow CNTs with unusual physical properties: Early theoretical calculations predicted that CNTs are either metals or semiconductors depending on atomic arrangements along their circumference. ${ }^{3-5)}$ Thus, CNTs are regarded as emerging materials for advancing further miniaturization of the electronic devices that is one of important demands in the semiconductor device technology. Indeed, it has been demonstrated that both an individual $\mathrm{CNT}^{6-8)}$ arrays of $\mathrm{CNTs}^{9,10)}$ and mat films of $\mathrm{CNTs}^{11,12)}$ work as conducting channels of field effect transistors (FETs) . According to the tubular structure of rigid C-C covalent bonds, CNTs have remarkable mechanical toughness, ${ }^{13,14}$ ) leading to the high thermal conductivity through them. ${ }^{15-17)}$ Therefore, CNTs are also regarded as the promising materials for designing composite materials and the energy harvesting devices, such as thermoelectric devices ${ }^{18,19)}$ and photovoltaic devices. ${ }^{20)}$

One-dimensional space with nanometer-scale diameter in CNTs allows an interesting class of compounds composed of host CNTs and encapsulated guest materials to be 
formed. In such host-guest compounds, guest materials form different condensed structures from those in their bulk states. Transmission electron microscopy experiments have confirmed that fullerenes and hydrocarbons form a one-dimensional chain inside CNTs. ${ }^{21-27)}$ Furthermore, because of the interaction between CNTs and guest materials, the electronic structures of these host-guest compounds are not the simple sum of those of the constituents. ${ }^{29-31)}$ These unusual nanometer-scale complexes have been regarded as one of the representative examples of van der Waals heterostructures whose structures are characterized by an interesting combination of one- and zero-dimensional constituent units, because all covalent bonds of them are perfectly saturated. In addition to the complexes consisting of CNT and molecules, CNTs can accommodate other one-dimensional materials, such as graphene nanoribbons ${ }^{25,26,28)}$ and other CNTs, ${ }^{32}$ ) being regarded as one-dimensional van der Waals heterostructures. Among such onedimensional complexes, double-walled CNTs are one of fascinating subject to study, because they exhibit peculiar low-energy electronic structures, caused by the curvature difference and inter-wall interaction. ${ }^{33-35)}$

Because of the structural similarity, boron nitride (BN) nanotube (BNNT) can form complexes where their constituents are coaxially stacked, just as the case of CNT. Furthermore, BNNT can form a coaxial complex with CNT and the complex can be used as an ultimate version of a one-dimensional semiconducting channel with a surrounded gate insulator, when a semiconducting CNT is wrapped by BNNT. The coaxial van der Waals heterostructures are a fascinating subject in the field of both nanoscience and nanotechnology. Despite several works address the electronic properties of the heterostructures with particular combinations, ${ }^{36,37)}$ there remains things unclear about their electronic structures such as band gaps of the CNT@BNNT when inner CNT is semiconductor. Thus, in this work, we aim to clarify the detailed energetics and electronic structures of BNNT encapsulating CNT (CNT@BNNT) in terms of inter-wall spacing and mutual atomic arrangement for providing the fundamentals of the coaxial heterostructures (Fig. 1), using the density functional theory (DFT). Our DFT calculations unveiled that the energetics of CNT@BNNT primarily depends on the inter-wall spacing between CNT and BNNT. In addition, we also found that the cohesion weakly depends on the mutual atomic arrangement between CNT and BNNT. The electronic structure of CNT near the band edges or the Fermi level strongly depends on the mutual atomic arrangement. By applying the external field, the carriers are injected into inner CNT, indicating that BNNT could work as a surrounded gate insulator for encapsulated 
CNT.

All the calculations were conducted using the $\mathrm{DFT}^{39,40)}$ as implemented in the Simulation Tool for Atom Technology (STATE) package. ${ }^{41)}$ To express the exchange correlation potential energy among interacting electrons, the local density approximation (LDA) is applied with the Perdew-Zunger functional form obtained by the quantum Monte Carlo calculations on a homogeneous electron gas, ${ }^{42,43)}$ because LDA qualitatively reproduces the weak interaction between nanoscale $\mathrm{sp}^{2} \mathrm{C}$ materials. ${ }^{44,45)} \mathrm{We}$ use an ultrasoft pseudopotential to describe the interactions between the valence electrons and ions generated by the Vanderbilt scheme. ${ }^{46)}$ The valence wavefunctions and deficit charge density were expanded with a plane-wave basis set with cutoff energies of 25 and $225 \mathrm{Ry}$, respectively. ${ }^{47)}$ For the Brillouin zone integration, six $k$-points were sampled along the tube axis. To inject the carriers into CNT@BNNT and to simulate CNT@BNNT-FET, we consider an FET with a planar metal electrode simulated by using the effective screening medium (ESM) method. ${ }^{48)}$

Double-walled nanotubes (CNT@BNNTs) consists of an inner zigzag CNT and an outer zigzag BNNT which are coaxially arranged with various mutual arrangements. We consider $(n, 0)$ CNT and $(m, 0)$ BNNT whose indices $n$ and $m$ range from $n=10$ to 16 and from $m=16$ to 25 , respectively. We impose the commensurability condition between lattice parameters of CNT and BNNT, where the lattice parameter of BNNT is shortened to match that of CNT. Geometric structures of CNT@BNNT are optimized under the fixed lattice parameter of $4.26 \AA$, until the force acting on atoms is less than $5 \mathrm{mRy} / \AA$. To investigate the energetics and electronic properties of an individual CNT@BNNT, the CNT@BNNT is separated from its periodic images by $9 \AA$-vacuum spacing. For the CNT@BNNT-FET, the gate electrode is located at $5 \AA$ above the outer wall of the CNT@BNNT.

The cohesive energy per atom between CNT and BNNT as a function of the interwall spacing, $\varepsilon$, is evaluated by

$$
\varepsilon=\frac{E_{\mathrm{CNT} @ \mathrm{BNNT}}-E_{\mathrm{CNT}}-E_{\mathrm{BNNT}}}{N_{\mathrm{C}}+N_{\mathrm{B}}+N_{\mathrm{N}}}
$$

where $E_{\mathrm{CNT} @ \mathrm{BNNT}}, E_{\mathrm{CNT}}$, and $E_{\mathrm{BNNT}}$ are the total energies of CNT@BNNT, CNT, and BNNT per unit cell, respectively, containing $N_{\mathrm{C}} \mathrm{C}$ atoms, $N_{\mathrm{B}} \mathrm{B}$ atoms, and $N_{\mathrm{N}}$ $\mathrm{N}$ atoms. As is shown in Fig. 2(a), the cohesive energy has minimum at $(10,0),(11,0)$, and $(12,0)$ CNTs for $(19,0),(20,0)$, and $(21,0)$ BNNTs, respectively, with the energy of approximately $10 \mathrm{meV} /$ atom, where the inter-wall spacing is $3.5 \AA$ being close to the 
interlayer spacings of graphite and hBN. ${ }^{49)}$

Figure 2(b) shows the cohesive energy between $(n, 0)$ CNT and $(n+9,0)$ BNNT with respect to their mutual inter-wall arrangements as a function of the tube diameter. Among three representative inter-wall arrangements, the $\mathrm{AB}(\mathrm{B})$ inter-wall arrangement, in which the axial position of $\mathrm{B}$ atoms are the same as $\mathrm{C}$ atoms, is the most stable configuration, irrespective of the chiral index $n$. On the other hand, the AA stacking where both $\mathrm{B}$ and $\mathrm{N}$ atoms have the same axial positions of $\mathrm{C}$ atoms is the least stable conformation for all CNT@BNNTs. The $\mathrm{AB}(\mathrm{N})$ arrangement, where the $\mathrm{N}$ atom is located above $\mathrm{C}$ atom with the same axial position, has the similar cohesive energy to that in the CNT@BNNT with the AA arrangement. In contrast to the inter-wall arrangement, the cohesive energy is insensitive to the tube diameter. Because of the incommensurability between inner and outer walls, inter-wall cohesion of CNT@BNNT with $\mathrm{AB}(\mathrm{B})$ and $\mathrm{AA}$ arrangements is weaker and stronger, respectively, than the interlayer cohesion between graphene and hBN with $\mathrm{AB}(\mathrm{B})$ and $\mathrm{AA}$ stacking arrangements.

Figure 3 shows the electronic structures of CNT@BNNT. For all cases, the electronic structures of CNT@BNNT are approximately the simple sums of those of the isolated CNT and BNNT. The (10,0) CNT@(19,0) BNNT is a semiconductor where band gap is the almost equivalent to that of the isolated (10,0) CNT [Fig. 3(a)]. The valence and conduction band edges of the CNT@BNNT are attributed from those of the isolated (10,0) CNT. Furthermore, the electronic energy band near the gap is approximately insensitive to the mutual sticking arrangement. For CNT@BNNT containing a metallic CNT, such as $(12,0)$ CNT@(21,0) BNNT showed the similar characteristics that CNT@BNNT with semiconducting CNT shows: The electronic energy bands near the Fermi level of $(12,0) \mathrm{CNT} @(21,0)$ BNNT almost retains those of an isolated $(12,0)$ CNT [Fig. 3(b)]. This result with inner metallic CNT is consistent with previous studies using DFT calculations. ${ }^{36,37)}$

We study the band gap of $(n, 0)$ CNT@ $(n+9,0)$ BNNT to give further insight into modulation of the electronic structure owing to their inter-wall interaction. Figure 4 shows the band gap of pristine $(n, 0)$ CNT and $(n, 0)$ CNT@ $(n+9,0)$ BNNT with AA, $\mathrm{AB}(\mathrm{N})$, and $\mathrm{AB}(\mathrm{B})$ stacking arrangements as a function of the tube index $n$. For $(n, 0)$ CNT@ $(n+9,0)$ BNNT with $\mathrm{AB}(\mathrm{B})$ mutual arrangement, the band gap is wider by 70 meV than that of the isolated $(n, 0)$ CNT with indices of $n=10$ and 13 , while it is narrower by $70 \mathrm{meV}$ than that of the isolated $(n, 0)$ CNT with indices of $n=11$ and 14. In contrast, the gap modulation is absent for the $(n, 0) \mathrm{CNT} @(n+9,0)$ BNNT with 
$\mathrm{AA}$ and $\mathrm{AB}(\mathrm{N})$ mutual atomic arrangements. These results indicate that encapsulated CNT effectively feels tensile strain along radial direction by BNNT under the AB(B) arrangement as the case of CNT encapsulating $\mathrm{C}_{60} \cdot{ }^{38)}$ The strain is ascribed to the relatively large interaction between $\mathrm{CNT}$ and $\mathrm{BNNT}$ with $\mathrm{AB}(\mathrm{B})$ arrangement. Furthermore, distribution of $\mathrm{B}$ wavefunction may also cause the peculiar gap modulation on CNT@BNNT with the AB(B) arrangement. It should be noted that the small but significant interaction between CNT and BNNT caused the small band gap for $(12,0)$ CNT@(21,0) BNNT and (15,0) CNT@(24,0) BNNT, when they are encapsulated in $(21,0)$ BNNT and $(24,0)$ BNNT, respectively. The electronic structure of CNT@BNNT shows that the electronic states near the band edges are ascribed to those of CNT, so that the BNNT acts as an insulating atomic layer for CNT, even though it slightly modulates the electronic structure of CNT. Thus, CNT@BNNT could be regarded as an ultimate version of the semiconducting/metallic wire surrounded by insulating layer.

Here, we calculate the carrier distribution and electrostatic potential of CNT@BNNT under the external electric field, to explore the ability of carrier accumulation in inner CNT. To inject carrier into CNT@BNNT, we consider the CNT@BNNTFET with a planar gate electrode simulated by an ideal metal metal with infinite permittivity, in which CNT@BNNT is located above the electrode with the $5.0 \AA$-vacuum spacing. Figure 5(a) and (b) show the contour plots of the carrier accumulation and depression in $(10,0)$ CNT@(19,0) BNNT under electron and hole doping, respectively, by the gate voltage. Injected carrier density is $+0.0704 e / \AA$ for electron doping and $0.0704 e / \AA$, where $e$ denotes elementary electricity. Carrier accumulation and depression is evaluated by

$$
\Delta \rho(\vec{r})=\rho_{\mathrm{F}}(\vec{r})-\rho_{0}(\vec{r})
$$

where $\rho_{\mathrm{F}}(\vec{r})$ and $\rho_{0}(\vec{r})$ correspond with the charge densities with and without the electric field. For both electron and hole doping, the polarization occurs at the wall of $(19,0)$ BNNT, reflecting its insulating nature, while electron/hole is accommodated into inner $(10,0)$ CNT. The carrier injection in CNT is corroborated by investigating the electrostatic potential modulation and electric field by the gate voltage [Figs. 5(c) and $5(\mathrm{~d})$ ]. Electrostatic potential of CNT is shallowest and deepest for electron and hole doping, respectively, with the radial electric field between CNT and BNNT. Therefore, CNT@BNNT is a potential material for nanoscale conducting channel with insulating layer. 
$\begin{array}{lll}\text { (a) } A A & \text { (b) } A B(N) \quad \text { (c) } A B(B)\end{array}$
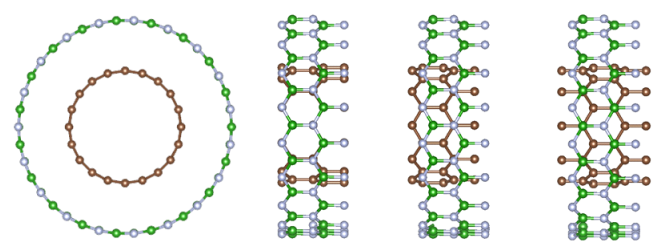

Fig. 1. Optimized structures of $(10,0) C N T @(19,0) B N N T$ with (a) $A A$, (b) $A B(N)$ and (c) $A B(B)$ inter-wall arrangements. Green, brown and cyan balls denote B, C and N atoms, respectively.

In summary, we study the energetics and electronic structures of CNT@BNNTs in terms of inter-wall spacing and mutual atomic arrangement for unraveling the fundamentals of coaxial van der Waals heterostructures, using the DFT with LDA. Our DFT-LDA calculations clarify that the energetics of CNT@BNNT primarily depend on the inter-wall spacing between CNT and BNNT. The largest cohesion occurs in the combination of $(n, 0)$ CNT and $(n+9,0)$ BNNT where the inter-wall spacing is approximately $3.5 \AA$. In addition to the inter-wall spacing, we also found that the cohesion weakly depends on the mutual atomic arrangement between CNT and BNNT. The electronic structure of CNT near the band edges or the Fermi level also weakly depends on the mutual atomic arrangement. For the CNT@BNNT with $\mathrm{AB}(\mathrm{B})$ inter-wall arrangement, inner CNT feels radial tensile strain by the BNNT, leading to the band gap modulation depending on their tube index. By applying the external field, the carriers are injected into inner CNT, indicating that BNNT could work as a surrounding tubular insulator for CNT.

\section{Acknowledgment}

Part of this work was financially supported by JSPS KAKENHI Grant Numbers JP15H05760, JP17K06187, JP18H05329 and JP19J13818. 
(a)

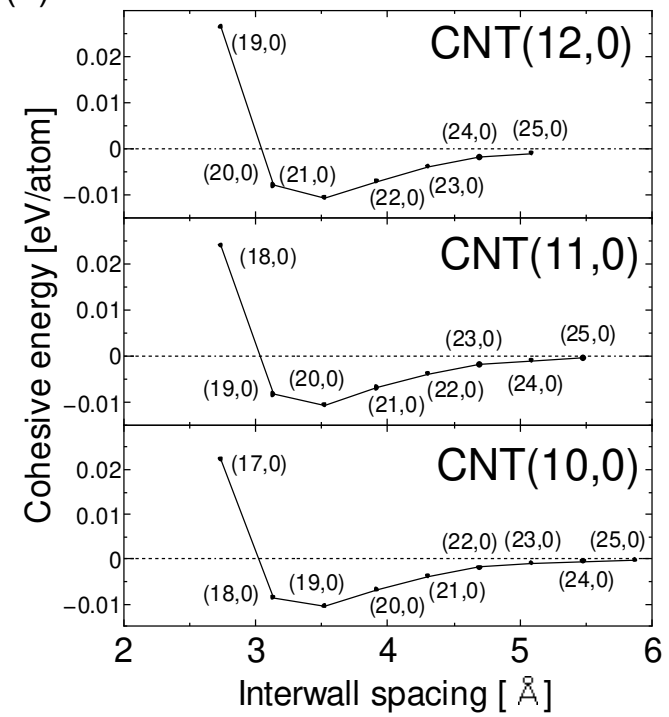

(b)

BNNT index $(m, 0)$

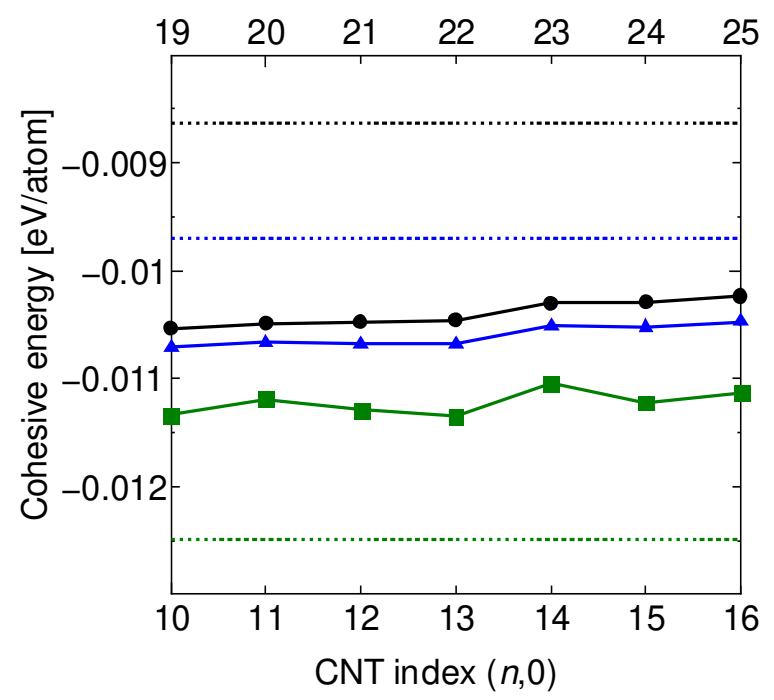

Fig. 2. (a) Cohesive energy of $(12,0),(11,0)$ and $(10,0)$ CNT in BNNT as a function of the inter-wall spacing. (b) Cohesive energy of $(n, 0)$ CNT@ $(m=n+9,0)$ BNNT as a function of the CNT index $n$. Circles, triangles and squares denote the energy of CNT@BNNT with $\mathrm{AA}, \mathrm{AB}(\mathrm{N})$ and $\mathrm{AB}(\mathrm{B})$ inter-wall arrangements, respectively. Cohesive energy between graphene and hBN with AA, $\mathrm{AB}(\mathrm{N})$ and $\mathrm{AB}(\mathrm{B})$ stacking arrangements are indicated by black, blue and green dotted lines, respectively. 
(a) $(10,0)$ CNT@(19,0) BNNT

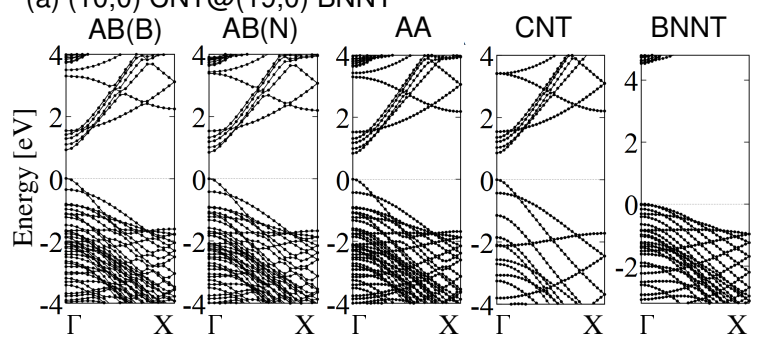

(b) $(12,0)$ CNT@(21,0) BNNT

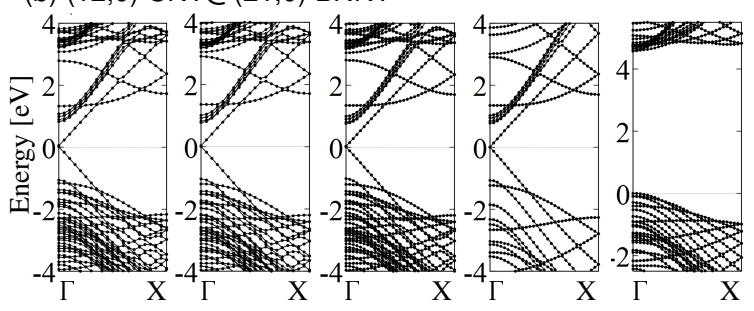

Fig. 3. (a) Electronic structure of $(10,0) \mathrm{CNT} @(19,0) \mathrm{BNNT}$ and (b) $(12,0) \mathrm{CNT} @(21,0) \mathrm{BNNT}$ with $\mathrm{AB}(\mathrm{B}), \mathrm{AB}(\mathrm{N})$ and $\mathrm{AA}$ inter-wall arrangements. In each figure, electronic structure of isolated CNT and BNNT are shown. The energy is measured from that of the valence band edge.

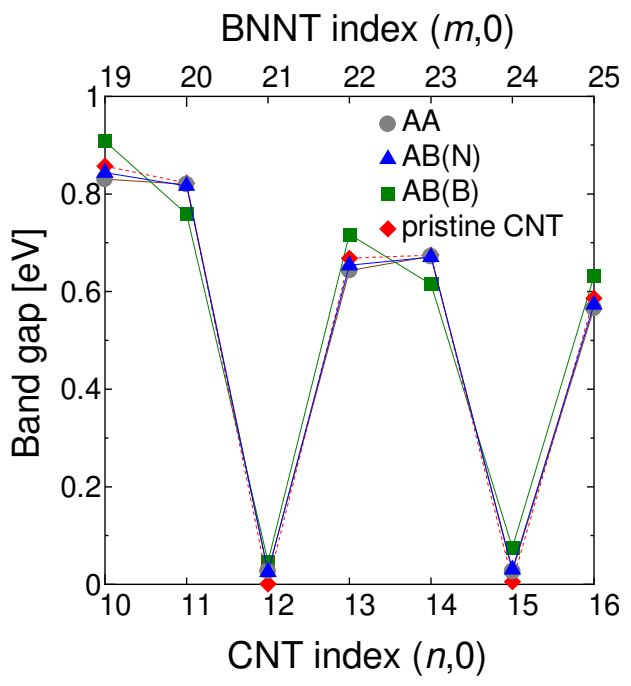

Fig. 4. Band gap of $(n, 0) \mathrm{CNT} @(m=n+9,0)$ BNNT as a function of the CNT index $n$. Circles, triangles and squares indicate the gap of CNT@BNNT with AA, AB(N) AB(B) inter-wall arrangements, respectively. Rhombuses indicate the band gap of isolated CNTs. 


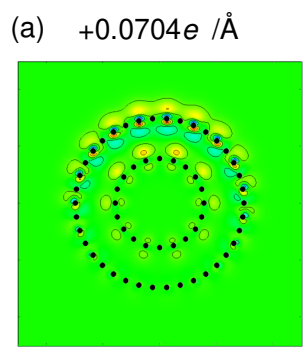

(b) $\quad-0.0704 e / \AA$

(c) $+0.0704 e / \AA$
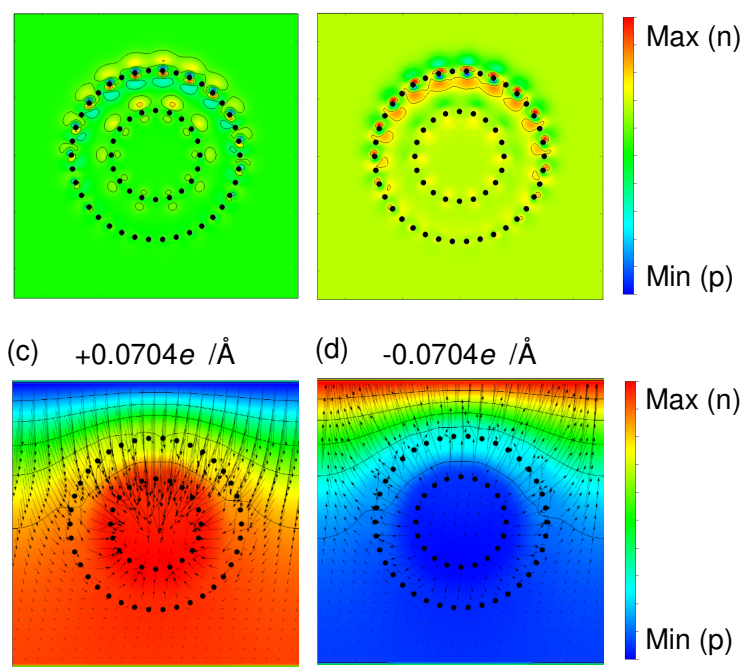

(d) $-0.0704 e / \AA$

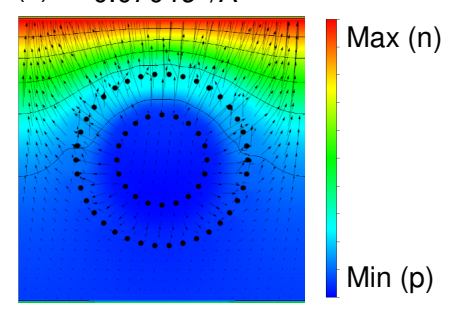

Fig. 5. Contour plots of the accumulated carriers in $(10,0) \mathrm{CNT} @(19,0)$ BNNT under (a) electron $(0.0704 e / \AA)$ and (b) hole $(-0.0704 e / \AA)$ doping. Contour and vector plots of electrostatic potential and electric field, respectively, of (c) electron and (d) hole doped $(10,0)$ CNT@(19,0) BNNT. Black circles indicate atomic positions of CNT and BNNT. 


\section{References}

1) S. Iijima, Nature 354, 56 (1991).

2) Single-Walled Carbon Nanotubes, edited by Y. Li and S. Maruyama (Springer, 2019)

3) N. Hamada, S. Sawada, and A. Oshiyama, Phys. Rev. Lett. 68, 1579 (1992).

4) R. Saito, M. Fujita, G. Dresselhaus, and M. S. Dresselhaus, Appl. Phys. Lett. 60, 2204 (1992).

5) K. Tanaka, K. Okahara, M. Okada, and T. Yamabe, Chem. Phys. Lett. 191, 469 (1992).

6) S. J. Tans, A. R. M. Verschueren, and C. Dekker, Nature 393, 49 (1998).

7) R. Martel, T. Schmidt, H. R. Shea, T. Hartel, and Ph. Avouris, Appl. Phys. Lett. 73, 2447 (1998).

8) C. Qiu, F. Liu, L. Xu, B. Deng, M. Xiao, J. Si, L. Lin, Z. Zhang, J. Wang, H. Guo, H. Peng, L. Peng, Science 361, 387 (2018).

9) G. Brady, A. Way, N. Safron, H. Evensen, P. Gopalan, and M. Arnold, Science Advances 2, 1601240 (2016).

10) G. Hills, C. Lau, A. Wright, S. Fuller, M. Bishop, T. Srimani, P. Kanhaiya, R. Ho, A. Amer, Y. Stein, D. Murphy, Arvind, A. Chandrakasan, and M. Shulaker Nature 572, 595 (2019).

11) D.-M. Sun, M. Y. Timmermans, Y. Tian, A. G. Nasibulin, E. I. Kauppinen, S. Kishimoto, T. Mizutani, and Y. Ohno, Nat. Nanotechnol. 6, 156 (2011).

12) C. Zhao, D. Zhong, J. Han, L. Liu, Z. Zhang, and L. Peng, Adv. Funct. Mater. 29, 1808574, (2019).

13) R. S. Ruoff and D. C. Lorents, Carbon 33, 925 (1995).

14) M. M. J. Treacy, T. W. Ebbesen, and J. M. Gibson, Nature 381, 678 (1996).

15) S. Berber, Y.-K. Kwon, and D. Tománek, Phys. Rev. Lett. 84, 4613 (2000).

16) T. Yamamoto, S. Watanabe, and K. Watanabe, Phys. Rev. Lett. 92, 075502 (2004).

17) S. Maruyama, Physica B 323, 193 (2002).

18) K. Yanagi, Appl. Phys. Express 9, 12503 (2016).

19) K. Fukuhara, Y. Ichinose, H. Nishidome, Y. Yomogida, F. Katsutani, N.

Komatsu, W. Gao, J. Kono, and K. Yanagi, Appl. Phys. Lett. 113, 243105 (2018)

20) I. Jeon, J. Yoon, U Kim, C. Lee, R. Xiang, A. Shawky, J. Xi, J. Byeon, H. Mo Lee, 
M. Choi, S. Maruyama, and Y. Matsuo, Adv. Energy Mater., 9, 1901204 (2019).

21) B. W. Smith, M. Monthioux, and D. E. Luzzi, Nature 396, 323 (1998).

22) B. Burteaux, A. Claye, B.W. Smith, M. Monthioux, D. E. Luzzi, and J. E. Fischer, Chem. Phys. Lett. 310, 21 (1999).

23) K. Hirahara, S. Bandow, K. Suenaga, H. Kato, T. Okazaki, H. Shinohara, and S. Iijima, Phys. Rev. B 64, 115420 (2001).

24) K. Hirahara, K. Suenaga, S. Bandow, H. Kato, T. Okazaki, H. Shinohara, and S. Iijima, Phys. Rev. Lett. 85, 5384 (2000).

25) M. Fujihara, Y. Miyata, R. Kitaura, Y. Nishimura, C. Camacho, S. Irle S, Y. Izumi, T. Okazaki, H. Shinohara, J. Phys. Chem. C 116, 15141 (2012).

26) H.E. Lim, Y. Miyata, M. Fujihara, S. Okada, H. Omachi, R. Kitaura, and H. Shinohara, ACS Nano 9, 5034 (2015).

27) T. Koyama, K. Fujiki, Y. Nagasawa, S. Okada, K. Asaka, Y. Saito, H. Kishida, J. Phys. Chem. C 122, 5805 ( 2018).

28) T. Okada, T. Kaneko, and R. Hatakeyama, Chem. Phys. Lett. 417, 289 (2006).

$29)$ S. Okada, S. Saito, and A. Oshiyama, Phys. Rev. Lett. 86, 3835 (2001).

30) M. Otani, S. Okada, and A. Oshiyama, Phys. Rev. B 68, 125424 (2003).

31) S. Okada, S. Saito, and A. Oshiyama, Phys. Rev. B 64, 201303(R) (2001).

32) P. M. Ajayan and S. Iijima, Nature 361, 333 (1993).

33) P. Delaney, H. J. Choi, J. Ihm, S. G. Louie, and M. L. Cohen, Nature, 391, 466 (1998).

34) Y.-K. Kwon, S. Saito, and D. Tománek, Phys. Rev. B, 58, R13314 (1998).

35) S. Okada and A. Oshiyama, Phys. Rev. Lett. 91, 216801 (2003).

36) Z. Zhang, W. Guo, and G. Tai, Appl. Phys. Lett. 90, 3 (2007).

37) A. Sengupta, J. Mater. Sci. 52, 8119 (2017).

38) T. Okazaki et al., J. Am. Chem. Soc. 130, 4122 (2008).

39) P. Hohenberg and W. Kohn, Phys. Rev. 136, B864 (1964).

40) W. Kohn and L. J. Sham, Phys. Rev. 140, A1133 (1965).

41) Y. Morikawa, K. Iwata, and K. Terakura, Appl. Surf. Sci. 169-170, 11 (2001).

42) J. P. Perdew and A. Zunger, Phys. Rev. B 23, 5048 (1981).

43) D. M. Ceperley and B. J. Alder, Phys. Rev. Lett. 45, 566 (1980).

44) S. Saito and A. Oshiyama, Phys. Rev. Lett. 66, 2637 (1991).

45) K. Kigure, Y. Iizumi, T. Okazaki, and S. Okada, J. Phys. Soc. Jpn. 83, 124709 (2014). 
46) D. Vanderbilt, Phys. Rev. B 41, 7892 (1990).

47) M. Maruyama and S. Okada, Carbon 125, 530 (2017).

48) M. Otani and O. Sugino, Phys. Rev. B 73, 115407 (2006).

49) S. Okada, Jpn. J. Appl. Phys. 49, 020204 (2010). 